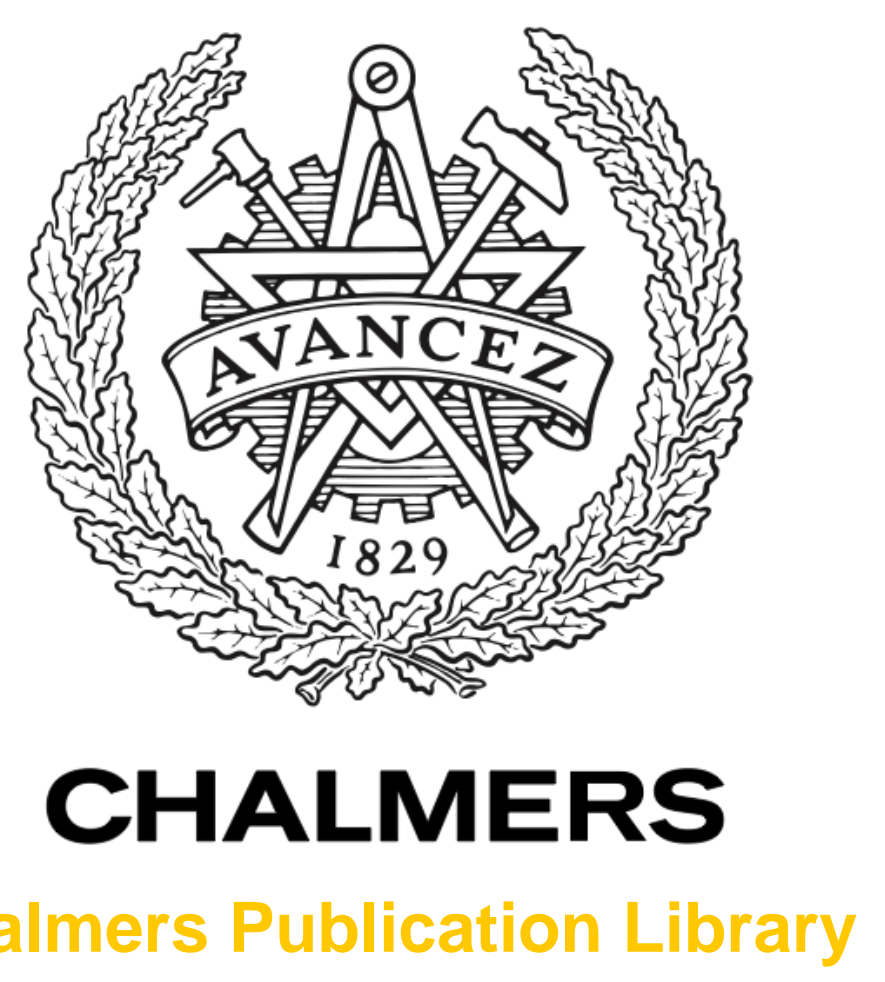

Chalmers Publication Library

Modelling of thin piezoelectric layers on plates

This document has been downloaded from Chalmers Publication Library (CPL). It is the author's version of a work that was accepted for publication in:

Wave motion (ISSN: 0165-2125)

Citation for the published paper:

Mauritsson, K. ; Boström, A. ; Folkow, P. (2008) "Modelling of thin piezoelectric layers on plates". Wave motion, vol. 45(5), pp. 616-628.

http://dx.doi.org/10.1016/j.wavemoti.2007.07.009

Downloaded from: http://publications.lib.chalmers.se/publication/73940

Notice: Changes introduced as a result of publishing processes such as copy-editing and formatting may not be reflected in this document. For a definitive version of this work, please refer to the published source. Please note that access to the published version might require a subscription.

Chalmers Publication Library (CPL) offers the possibility of retrieving research publications produced at Chalmers University of Technology. It covers all types of publications: articles, dissertations, licentiate theses, masters theses, conference papers, reports etc. Since 2006 it is the official tool for Chalmers official publication statistics. To ensure that Chalmers research results are disseminated as widely as possible, an Open Access Policy has been adopted.

The CPL service is administrated and maintained by Chalmers Library. 


\title{
MODELLING OF THIN PIEZOELECTRIC LAYERS ON PLATES
}

\author{
K. MAURITSSON, A. BOSTRÖM, P.D. FOLKOW
}

\begin{abstract}
The derivation of plate equations for a plate consisting of two layers, one anisotropic elastic and one piezoelectric, is considered. Power series expansions in the thickness coordinate for the displacement components and the electric potential lead to recursion relations among the expansion functions. Using these in the boundary and interface conditions, a set of equations is obtained for some of the lowest-order expansion functions. This set is reduced to six equations corresponding to the symmetric (in-plane) and antisymmetric (bending) motions of the elastic layer. These equations are given to linear (for the symmetric equations) or quadratic (for the antisymmetric equations) order in the thickness. It is noted that it is, in principle, possible to go to any order, and that it is believed that the corresponding equations are asymptotically correct. A few numerical results for guided waves along the plate and a 1D actuator case illustrate the accuracy.
\end{abstract}

\section{INTRODUCTION}

The use of piezoelectric patches or layers for applications in 'smart' structures has been considered in recent years. In these cases the thickness of the piezoelectric layer is usually thin (in comparison to relevant wavelengths) as is the structure to which it is attached. It is therefore natural to develop beam, plate and shell equations for layered structures including piezoelectric layers. The review articles by Gopinathan et al. [1] and Wang and Yang [2] give many references to work prior to 2000. In recent years many investigations concern the derivation of plate and shell equations, but only a few references are given here $[3,4,5,6,7]$.

In this paper, the attention is on the derivation of plate equations for a layered plate including also piezoelectric layers. This is accomplished by expanding all field quantities in the layers in power series in the thickness coordinate, to arbitrary order in principle. Insertion into the equations of motion gives recursion relations among the series coefficients that can be used to eliminate all but some of the lowest-order coefficients. The boundary and interface conditions then give a set of equations for these coefficients, where various eliminations and truncations can be performed. All lengthy analytical calculations involved are performed with Mathematica. This approach has previously been adopted for an isotropic plate, see Boström et al. [8], and for deriving equivalent boundary conditions for a thin piezoelectric layer, see Johansson and Niklasson [9], Zhang et al. [10], and Boström and Zhang [11].

To be specific, a plate with two layers is chosen according to Fig. 1. The upper piezoelectric layer has thickness $h_{\mathrm{p}}$ and the lower elastic one has thickness $h_{\mathrm{e}}$. The piezoelectric layer is assumed to be of class $2 \mathrm{~mm}$ poled in the normal direction and the elastic layer is orthotropic. This covers many material combinations of practical interest, in particular polyvinylidene fluoride (PVDF) and piezocheramics (PZT) (of subclass $6 \mathrm{~mm}$ ). It will 


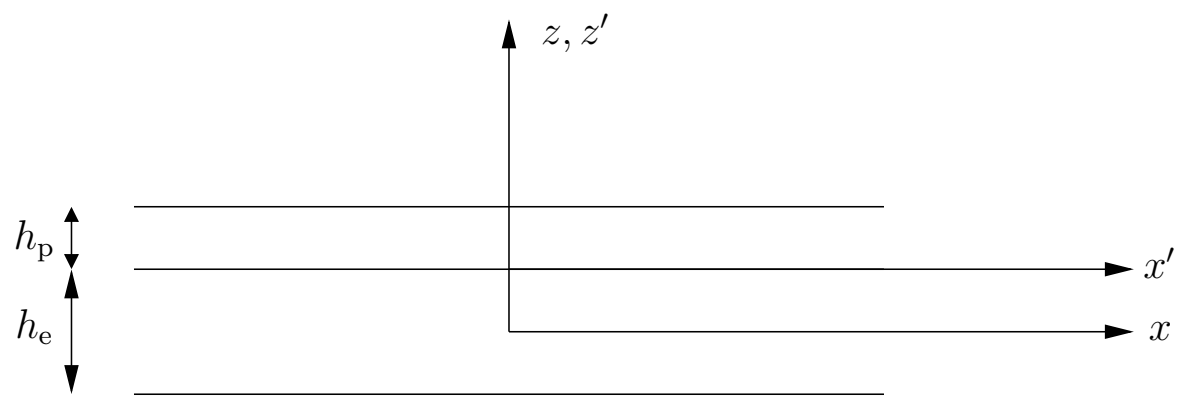

FiguRE 1. The geometry with a two-layered plate and two coordinate systems.

prove convenient to use two vertical axes $z$ and $z^{\prime}$ as shown in Fig. 1, one with origin in the middle of the elastic layer and one with origin at the interface. The focus is on deriving plate equations using series expansions in the thickness direction and thus only an infinite plate without boundary conditions along any edges of the plate is treated. It is noted that there are good reasons to believe that the present approach leads to asymptotically correct equations, in principle to any order [8].

\section{BASIC EQUATIONS}

In this section the basic equations in a linear piezoelectric material are briefly reviewed. The material is assumed to be of class $2 \mathrm{~mm}$ and elastically it is thus orthotropic. As is common the quasistatic approximation is made and thus no magnetic fields are involved. It is noted that this approximation makes the theory nonhyperbolic. The notation follows that of Auld [12]. Thus, standard tensor notation is used. The mechanical fields in the piezoelectric layer are given an upper index 'p' to distinguish them from the corresponding quantities in the elastic layer. The basic equations governing the motion in a piezoelectric continuum are

$$
\begin{aligned}
T_{i j, i}^{\mathrm{p}} & =\rho_{\mathrm{p}} \ddot{u}_{j}^{\mathrm{p}}, \\
D_{i, i} & =0 .
\end{aligned}
$$

Here $T_{i j}^{\mathrm{p}}$ is the stress tensor, $u_{i}^{\mathrm{p}}$ is the displacement vector, $D_{i}$ is the electric displacement vector, and $\rho_{\mathrm{p}}$ is the density. The constitutive equations in a linear piezoelectric material are

$$
\begin{aligned}
T_{i j}^{\mathrm{p}} & =k_{i j k l} S_{k l}^{\mathrm{p}}-e_{k i j} E_{k}, \\
D_{i} & =e_{i k l} S_{k l}^{\mathrm{p}}+\epsilon_{i k} E_{k},
\end{aligned}
$$

where $S_{i j}^{\mathrm{p}}$ is the strain tensor, $E_{i}$ is the electric field vector, $k_{i j k l}$ is the elastic stiffness tensor, $\epsilon_{i j}$ is the dielectric tensor, and $e_{i j k}$ is the piezoelectric coupling tensor. The nonstandard notation for the stiffness tensor is used to avoid an awkward upper index 'p' in the lengthy expression that will later involve the stiffness constants. The standard notation $c_{i j k l}$ is used for the stiffness tensor in the elastic layer.

The strain is given in terms of the displacement

$$
S_{i j}^{\mathrm{p}}=\frac{1}{2}\left(u_{i, j}^{\mathrm{p}}+u_{j, i}^{\mathrm{p}}\right) .
$$


In the quasistatic approximation the electric field is given by the gradient of the electric potential

$$
E_{i}=-\Phi_{, i}
$$

Equations (2.1)-(2.6) can of course be combined to obtain the governing equations for the displacement $u_{i}^{\mathrm{p}}$ and the electric potential $\Phi$

$$
\begin{aligned}
& k_{i j k l} u_{k, l i}^{\mathrm{p}}+e_{k i j} \Phi_{, k i}=\rho_{\mathrm{p}} \ddot{u}_{j}^{\mathrm{p}}, \\
& e_{i k l} u_{k, l i}^{\mathrm{p}}-\epsilon_{i k} \Phi_{, k i}=0 .
\end{aligned}
$$

Here it is clearly seen that the piezoelectric coupling tensor $e_{i j k}$ gives the coupling between the mechanical and electric fields.

As the piezoelectric layer is of class $2 \mathrm{~mm}$ it is orthotropic with nine independent stiffness constants (using abbreviated subscripts, see Auld [12])

$$
\left(k_{I J}\right)=\left(\begin{array}{cccccc}
k_{11} & k_{12} & k_{13} & 0 & 0 & 0 \\
k_{12} & k_{22} & k_{23} & 0 & 0 & 0 \\
k_{13} & k_{23} & k_{33} & 0 & 0 & 0 \\
0 & 0 & 0 & k_{44} & 0 & 0 \\
0 & 0 & 0 & 0 & k_{55} & 0 \\
0 & 0 & 0 & 0 & 0 & k_{66}
\end{array}\right)
$$

There are five independent piezoelectric coupling constants

$$
\left(e_{i J}\right)=\left(\begin{array}{cccccc}
0 & 0 & 0 & 0 & e_{x 5} & 0 \\
0 & 0 & 0 & e_{y 4} & 0 & 0 \\
e_{z 1} & e_{z 2} & e_{z 3} & 0 & 0 & 0
\end{array}\right),
$$

and three independent dielectric constants

$$
\left(\epsilon_{i j}\right)=\left(\begin{array}{ccc}
\epsilon_{x x} & 0 & 0 \\
0 & \epsilon_{y y} & 0 \\
0 & 0 & \epsilon_{z z}
\end{array}\right)
$$

Note that the ordinary vector indices are denoted $i=x, y, z$, whereas the abbreviated indices are $I=1,2,3,4,5,6$.

The governing equations in the elastic layer can be obtained as a special case of the above with the piezoelectric coupling tensor put to zero and no electric fields present. As noted above the stiffness tensor in the elastic layer is denoted $c_{i j k l}$ and the density is $\rho_{\mathrm{e}}$.

\section{SERIES EXPANSIONS}

To derive plate equations for the layered plate the displacement components and the electric potential are now expanded in series in the thickness coordinate. This easily gives recursion relations among the expansion coefficients so that all but those two of lowest order can be eliminated. Insertion into the boundary and interface conditions then gives a set of equations for the remaining expansion coefficients. These can be further processed in different ways.

When performing the series expansions the origin for the expansions can be put at different points and various choices have their merits. Putting the origin at the interface between 
the two layers leads to great simplifications in the interface conditions. For the present case with a piezoelectric layer on top of an elastic one, it will often be the case that the piezoelectric layer is in the form of a smaller patch that only covers a part of an elastic plate, and outside the piezoelectric patch it is then natural to use a plate equation with the origin in the middle (in the thickness direction). It is also better to put the origin in the middle because this leads to an approximate decoupling into symmetric (in-plane) and antisymmetric (bending) motion and, furthermore, a plate equation gives a better approximation with the origin in the middle. For these reasons the origin for the elastic layer is chosen in the middle of the layer, see Fig. 1 for the coordinate systems. The origin for the piezoelectric layer is chosen at the interface because this leads to great simplifications in the interface conditions. In the piezoelectric layer the expansions are thus

$$
\begin{aligned}
u^{\mathrm{p}}\left(x, y, z^{\prime}, t\right) & =\sum_{j=0}^{\infty} u_{j}^{\mathrm{p}}(x, y, t)\left(z^{\prime}\right)^{j}, \\
v^{\mathrm{p}}\left(x, y, z^{\prime}, t\right) & =\sum_{j=0}^{\infty} v_{j}^{\mathrm{p}}(x, y, t)\left(z^{\prime}\right)^{j}, \\
w^{\mathrm{p}}\left(x, y, z^{\prime}, t\right) & =\sum_{j=0}^{\infty} w_{j}^{\mathrm{p}}(x, y, t)\left(z^{\prime}\right)^{j}, \\
\Phi\left(x, y, z^{\prime}, t\right) & =\sum_{j=0}^{\infty} \Phi_{j}(x, y, t)\left(z^{\prime}\right)^{j},
\end{aligned}
$$

where the $z^{\prime}$ axis is thus chosen so that $z^{\prime}=0$ at the interface. To avoid double indices, a change in notation is made to $u^{\mathrm{p}}, v^{\mathrm{p}}, w^{\mathrm{p}}$, for the three displacement components. In the elastic layer the displacement components are similarly expanded with expansion functions $u_{j}, v_{j}, w_{j}$, but as stressed above the origin for these expansions are chosen in the middle of the elastic layer at $z=0$.

The power series expansions Eqs. (3.1)-(3.4) are now inserted into the governing equations (2.7) and (2.8) and as the resulting equations are valid for all $z^{\prime}$ the coefficients of each power in the series can be put equal to zero individually. It is then straightforward to obtain the following recursion relations among the coefficients:

$$
\begin{aligned}
u_{j+2}^{\mathrm{p}}= & \frac{1}{(j+1)(j+2) k_{55}}\left[\rho_{\mathrm{p}} \partial_{t}^{2} u_{j}^{\mathrm{p}}-k_{11} \partial_{x}^{2} u_{j}^{\mathrm{p}}-k_{66} \partial_{y}^{2} u_{j}^{\mathrm{p}}-k_{126} \partial_{x} \partial_{y} v_{j}^{\mathrm{p}}\right. \\
& \left.-(j+1) k_{135} \partial_{x} w_{j+1}^{\mathrm{p}}-(j+1) e_{51} \partial_{x} \Phi_{j+1}\right], \\
v_{j+2}^{\mathrm{p}}= & \frac{1}{(j+1)(j+2) k_{44}}\left[\rho_{\mathrm{p}} \partial_{t}^{2} v_{j}^{\mathrm{p}}-k_{22} \partial_{y}^{2} v_{j}^{\mathrm{p}}-k_{66} \partial_{x}^{2} v_{j}^{\mathrm{p}}-k_{126} \partial_{x} \partial_{y} u_{j}^{\mathrm{p}}\right. \\
& \left.-(j+1) k_{234} \partial_{y} w_{j+1}^{\mathrm{p}}-(j+1) e_{42} \partial_{y} \Phi_{j+1}\right], \\
w_{j+2}^{\mathrm{p}}= & \frac{1}{(j+1)(j+2) h_{33}}\left[\epsilon_{z z} \rho_{\mathrm{p}} \partial_{t}^{2} w_{j}^{\mathrm{p}}-h_{55} \partial_{x}^{2} w_{j}^{\mathrm{p}}-h_{44} \partial_{y}^{2} w_{j}^{\mathrm{p}}\right. \\
& \left.-(j+1) h_{135} \partial_{x} u_{j+1}^{\mathrm{p}}-(j+1) h_{234} \partial_{y} v_{j+1}^{\mathrm{p}}-f_{53} \partial_{x}^{2} \Phi_{j}-f_{43} \partial_{y}^{2} \Phi_{j}\right],
\end{aligned}
$$




$$
\begin{aligned}
\Phi_{j+2}= & \frac{1}{(j+1)(j+2) h_{33}}\left[e_{z 3} \rho_{\mathrm{p}} \partial_{t}^{2} w_{j}^{\mathrm{p}}-g_{53} \partial_{x}^{2} w_{j}^{\mathrm{p}}-g_{43} \partial_{y}^{2} w_{j}^{\mathrm{p}}\right. \\
& \left.-(j+1) g_{135} \partial_{x} u_{j+1}^{\mathrm{p}}-(j+1) g_{234} \partial_{y} v_{j+1}^{\mathrm{p}}-h_{35} \partial_{x}^{2} \Phi_{j}-h_{34} \partial_{y}^{2} \Phi_{j}\right],
\end{aligned}
$$

where the new material constants that are introduced are combinations of the original ones, see below. The last two equations are obtained as the solution of a system of two equations. It is noted that Eqs. (3.5)-(3.8) can be used to express all the $u_{j}^{\mathrm{p}}, v_{j}^{\mathrm{p}}, w_{j}^{\mathrm{p}}, \Phi_{j}$, for $j=2,3, \ldots$, in terms of the eight lowest order ones for $j=0,1$. The new constants in Eqs. (3.5)-(3.8) are

$$
\begin{array}{lll}
k_{126}=k_{12}+k_{66}, & k_{135}=k_{13}+k_{55}, & k_{234}=k_{23}+k_{44}, \\
e_{51}=e_{x 5}+e_{z 1}, & e_{42}=e_{y 4}+e_{z 2}, & \\
h_{33}=k_{33} \epsilon_{z z}+e_{z 3}^{2}, & h_{44}=k_{44} \epsilon_{z z}+e_{y 4} e_{z 3}, & h_{55}=k_{55} \epsilon_{z z}+e_{x 5} e_{z 3}, \\
h_{34}=k_{33} \epsilon_{y y}+e_{y 4} e_{z 3}, & h_{35}=k_{33} \epsilon_{x x}+e_{x 5} e_{z 3}, & \\
h_{135}=k_{135} \epsilon_{z z}+e_{51} e_{z 3}, & h_{234}=k_{234} \epsilon_{z z}+e_{42} e_{z 3}, & \\
f_{43}=e_{y 4} \epsilon_{z z}-e_{z 3} \epsilon_{y y}, & f_{53}=e_{x 5} \epsilon_{z z}-e_{z 3} \epsilon_{x x}, \\
g_{43}=k_{44} e_{z 3}-k_{33} e_{y 4}, & g_{53}=k_{55} e_{z 3}-k_{33} e_{x 5}, & \\
g_{135}=k_{135} e_{z 3}-k_{33} e_{51}, & g_{234}=k_{234} e_{z 3}-k_{33} e_{42} .
\end{array}
$$

The notation partly reflects the nature of the constants; thus all constants with the same letter but different indices have the same dimension and the indices more or less reflect the indices of the underlying material constants.

For the elastic layer the series expansions and recursion relations are of course very similar; it is just to drop $\Phi$ and put all piezoelectric coupling constants equal to zero.

To continue, the boundary and interface conditions must now be applied. At the free surface of the elastic layer at $z=-h_{\mathrm{e}} / 2$ the stress vector vanishes. Inserting the series expansions this gives

$$
\begin{aligned}
& T_{z z}=\sum_{j=0}^{\infty}\left[c_{13} \partial_{x} u_{j}+c_{23} \partial_{y} v_{j}+(j+1) c_{33} w_{j+1}\right]\left(-h_{\mathrm{e}} / 2\right)^{j}=0, \\
& T_{x z}=\sum_{j=0}^{\infty} c_{55}\left[(j+1) u_{j+1}+\partial_{x} w_{j}\right]\left(-h_{\mathrm{e}} / 2\right)^{j}=0, \\
& T_{y z}=\sum_{j=0}^{\infty} c_{44}\left[(j+1) v_{j+1}+\partial_{y} w_{j}\right]\left(-h_{\mathrm{e}} / 2\right)^{j}=0,
\end{aligned}
$$

where for better clarity the derivatives are denoted $\partial_{x}$ and $\partial_{y}$ instead of with a comma. 
The stress free boundary condition at the top of the piezoelectric layer at $z^{\prime}=h_{\mathrm{p}}$ similarly gives

$$
\begin{aligned}
T_{z z}^{\mathrm{p}} & =\sum_{j=0}^{\infty}\left[k_{13} \partial_{x} u_{j}^{\mathrm{p}}+k_{23} \partial_{y} v_{j}^{\mathrm{p}}+(j+1) k_{33} w_{j+1}^{\mathrm{p}}+(j+1) e_{z 3} \Phi_{j+1}\right] h_{\mathrm{p}}^{j}=0, \\
T_{x z}^{\mathrm{p}} & =\sum_{j=0}^{\infty}\left[(j+1) k_{55} u_{j+1}^{\mathrm{p}}+k_{55} \partial_{x} w_{j}^{\mathrm{p}}+e_{x 5} \partial_{x} \Phi_{j}\right] h_{\mathrm{p}}^{j}=0, \\
T_{y z}^{\mathrm{p}} & =\sum_{j=0}^{\infty}\left[(j+1) k_{44} v_{j+1}^{\mathrm{p}}+k_{44} \partial_{y} w_{j}^{\mathrm{p}}+e_{y 4} \partial_{y} \Phi_{j}\right] h_{\mathrm{p}}^{j}=0 .
\end{aligned}
$$

The interface conditions involve the continuity of displacement and stress. The displacement condition at the interface $\left(z=h_{\mathrm{e}} / 2, z^{\prime}=0\right)$ gives

$$
\begin{aligned}
& u_{0}^{\mathrm{p}}=\sum_{j=0}^{\infty} u_{j}\left(h_{\mathrm{e}} / 2\right)^{j}, \\
& v_{0}^{\mathrm{p}}=\sum_{j=0}^{\infty} v_{j}\left(h_{\mathrm{e}} / 2\right)^{j}, \\
& w_{0}^{\mathrm{p}}=\sum_{j=0}^{\infty} w_{j}\left(h_{\mathrm{e}} / 2\right)^{j},
\end{aligned}
$$

and the stress condition gives (for the $z, x$ and $y$ components, respectively)

$$
\begin{aligned}
& k_{13} \partial_{x} u_{0}^{\mathrm{p}}+k_{23} \partial_{y} v_{0}^{\mathrm{p}}+k_{33} w_{1}^{\mathrm{p}}+e_{z 3} \Phi_{1} \\
& =\sum_{j=0}^{\infty}\left[c_{13} \partial_{x} u_{j}+c_{23} \partial_{y} v_{j}+(j+1) c_{33} w_{j+1}\right]\left(h_{\mathrm{e}} / 2\right)^{j}, \\
& k_{55} u_{1}^{\mathrm{p}}+k_{55} \partial_{x} w_{0}^{\mathrm{p}}+e_{x 5} \partial_{x} \Phi_{0}=\sum_{j=0}^{\infty} c_{55}\left[(j+1) u_{j+1}+\partial_{x} w_{j}\right]\left(h_{\mathrm{e}} / 2\right)^{j}, \\
& k_{44} v_{1}^{\mathrm{p}}+k_{44} \partial_{y} w_{0}^{\mathrm{p}}+e_{y 4} \partial_{y} \Phi_{0}=\sum_{j=0}^{\infty} c_{44}\left[(j+1) v_{j+1}+\partial_{y} w_{j}\right]\left(h_{\mathrm{e}} / 2\right)^{j} .
\end{aligned}
$$

There remains to specify the electric boundary conditions. These depend on whether the piezoelectric layer is used as an actuator or sensor. For the actuator case the electric potential is specified at the electrodes at the bottom and top of the layer

$$
\begin{aligned}
& \Phi_{0}=V_{0}, \\
& \sum_{j=0}^{\infty} \Phi_{j}\left(h_{\mathrm{p}}\right)^{j}=V_{1} .
\end{aligned}
$$

Here the applied potential $V_{0}$ at the interface and $V_{1}$ at the top of the piezoelectric layer may be functions of time but not of the space coordinates. If the electrodes are short-circuited $V_{0}=V_{1}$. 


\section{Plate equations}

As noted above the recursion relations can be used to eliminate all expansion functions with index $j=2,3, \ldots$. In this way Eqs. (3.10)-(3.23) constitute 14 differential equations in 14 unknown functions, namely those with index $j=0$ and $j=1$ in the two layers (including the electric potential in the piezoelectric layer). Due to the location of the origin for the expansion in the piezoelectric layer, Eqs. (3.16)-(3.22) can easily be solved for $u_{0}^{\mathrm{p}}, u_{1}^{\mathrm{p}}, v_{0}^{\mathrm{p}}$, $v_{1}^{\mathrm{p}}, w_{0}^{\mathrm{p}}, w_{1}^{\mathrm{p}}$, and $\Phi_{0}$. There then remain seven equations in the displacement functions in the elastic layer $u_{0}, u_{1}, v_{0}, v_{1}, w_{0}$ and $w_{1}$ and the electric potential expansion function $\Phi_{1}$. It is noted that the manipulations leading to these seven equations only involve substitutions that can, in principle, be carried out to any order. Thus, no truncations or approximations have been performed so far.

In the seven equations it is very natural to eliminate $\Phi_{1}$ as this is the only expansion function referring to the piezoelectric layer. It should, however, be noted that a reduction to six or less equations has the drawback that it is no longer simple to obtain the displacement and other fields in the piezoelectric layer. In many cases these fields are of no direct interest, so a reduction to six equations are performed here (but an exception is made below when the fields in the piezoelectric layer are computed for guided waves).

There then remain six equations and there are of course ways to reduce the number of equations further, but this results in extremely lengthy equations, so only a reduction to six equations is performed here. By using the recursion relations all the expansion functions can then be obtained and thus also the stresses, but this is not possible in a straightforward way if fewer equations are employed. Furthermore, it resembles common practice for higher-order equations when no mirror symmetry is present in the plate and thus no division into symmetric and antisymmetric parts is possible. However, if the piezoelectric layer is thin compared to the elastic one, an approximate division into these two parts is possible. When $\Phi_{1}$ is eliminated with the help of Eq. (3.23) the remaining six equations are added and subtracted pairwise to obtain equations that decouple into symmetric and antisymmetric groups in the limit when the thickness of the piezoelectric layer vanishes.

When all these lengthy manipulations have been performed the three equations corresponding to the symmetric (in the limit of vanishing piezoelectric layer) part become

$$
\begin{gathered}
-\bar{\rho} \partial_{t}^{2} u_{0}+\bar{c}_{66} \partial_{y}^{2} u_{0}+\left(\bar{c}_{11}+\alpha \gamma_{13} c_{13}^{\star}\right) \partial_{x}^{2} u_{0} \\
+\left(\bar{c}_{126}+\alpha \gamma_{13} c_{23}^{\star}\right) \partial_{x} \partial_{y} v_{0}+\left(c_{13}+\alpha \gamma_{13} c_{33}\right) \partial_{x} w_{1} \\
+\frac{1}{2} \alpha h_{\mathrm{e}}\left[\hat{\gamma}_{55}\left(-\rho_{\mathrm{p}} \partial_{t}^{2} u_{1}+k_{66} \partial_{y}^{2} u_{1}\right)+\left(\hat{\gamma}_{55} k_{113}-(1+\alpha) \gamma_{13} c_{55}\right) \partial_{x}^{2} u_{1}\right. \\
+\left(\hat{\gamma}_{44}\left(k_{126}-\gamma_{13} k_{23}\right)-(1+\alpha) \gamma_{13} c_{44}\right) \partial_{x} \partial_{y} v_{1} \\
+\left(\gamma_{13} \bar{\rho}-\alpha \gamma_{55}^{\star} \rho_{\mathrm{p}}\right) \partial_{x} \partial_{t}^{2} w_{0}+\left(\alpha k_{113} \gamma_{55}^{\star}-(1+\alpha) \gamma_{13} c_{55}\right) \partial_{x}^{3} w_{0} \\
\left.+\left(\alpha \gamma_{44}^{\star}\left(k_{126}-\gamma_{13} k_{23}\right)+\alpha \gamma_{55}^{\star} k_{66}-(1+\alpha) \gamma_{13} c_{44}\right) \partial_{x} \partial_{y}^{2} w_{0}\right]=0
\end{gathered}
$$




$$
\begin{gathered}
-\bar{\rho} \partial_{t}^{2} v_{0}+\bar{c}_{66} \partial_{x}^{2} v_{0}+\left(\bar{c}_{22}+\alpha \gamma_{23} c_{23}^{\star}\right) \partial_{y}^{2} v_{0} \\
+\left(\bar{c}_{126}+\alpha \gamma_{23} c_{13}^{\star}\right) \partial_{x} \partial_{y} u_{0}+\left(c_{23}+\alpha \gamma_{23} c_{33}\right) \partial_{y} w_{1} \\
+\frac{1}{2} \alpha h_{\mathrm{e}}\left[\hat{\gamma}_{44}\left(-\rho_{\mathrm{p}} \partial_{t}^{2} v_{1}+k_{66} \partial_{x}^{2} v_{1}\right)+\left(\hat{\gamma}_{44} k_{223}-(1+\alpha) \gamma_{23} c_{44}\right) \partial_{y}^{2} v_{1}\right. \\
+\left(\hat{\gamma}_{55}\left(k_{126}-\gamma_{23} k_{13}\right)-(1+\alpha) \gamma_{23} c_{55}\right) \partial_{x} \partial_{y} u_{1} \\
+\left(\gamma_{23} \bar{\rho}-\alpha \gamma_{44}^{\star} \rho_{\mathrm{p}}\right) \partial_{y} \partial_{t}^{2} w_{0}+\left(\alpha k_{223} \gamma_{44}^{\star}-(1+\alpha) \gamma_{23} c_{44}\right) \partial_{y}^{3} w_{0} \\
\left.+\left(\alpha \gamma_{55}^{\star}\left(k_{126}-\gamma_{23} k_{13}\right)+\alpha \gamma_{44}^{\star} k_{66}-(1+\alpha) \gamma_{23} c_{55}\right) \partial_{x}^{2} \partial_{y} w_{0}\right]=0 \\
c_{13} \partial_{x} u_{0}+c_{23} \partial_{y} v_{0}+c_{33} w_{1}+\frac{\alpha h_{\mathrm{e}}}{2}\left[\rho_{\mathrm{p}} \partial_{t}^{2} w_{0}-c_{55} \partial_{x}^{2} w_{0}\right. \\
\left.-c_{44} \partial_{y}^{2} w_{0}-c_{55} \partial_{x} u_{1}-c_{44} \partial_{y} v_{1}\right]=\frac{\alpha h_{\mathrm{e}} e_{z 3} \rho_{\mathrm{p}}}{4 k_{33}} \partial_{t}^{2} \Delta V
\end{gathered}
$$

Here only terms linear in $h_{\mathrm{e}}$ are retained. $\Delta V=V_{1}-V_{0}$ is the applied electric potential difference across the piezoelectric layer. The constant $\alpha=h_{\mathrm{p}} / h_{\mathrm{e}}$ measures the relative thickness of the piezoelectric layer compared to the elastic one. Some new constants are introduced and as the expressions become rather lengthy these are defined in Appendix A. It is worth mentioning that an overbar essentially indicates a mean through the whole plate of the corresponding density or stiffness. A star indicates a difference and the constants $\gamma$ with indices denote dimensionless stiffnesses.

Equation (4.3) is a modified plane stress condition which contains also a source term from the applied voltage. For a thin piezoelectric layer (small $\alpha$ ) this term is expected to be small. The other two equations are essentially describing the $2 \mathrm{D}$ in-plane motion modified with the linear terms in $h_{\mathrm{e}}$ to account for the coupling to the bending motion.

The three equations corresponding to the antisymmetric (in the limit of vanishing piezoelectric layer) part are

$$
\begin{gathered}
c_{55}\left(u_{1}+\partial_{x} w_{0}\right)+\frac{\alpha h_{\mathrm{e}}}{2}\left[\rho_{\mathrm{p}} \partial_{t}^{2} u_{0}-k_{66} \partial_{y}^{2} u_{0}-\left(k_{11}+\gamma_{13} c_{13}^{\star}\right) \partial_{x}^{2} u_{0}\right. \\
\left.-\left(k_{126}+\gamma_{13} c_{23}^{\star}\right) \partial_{x} \partial_{y} v_{0}-\gamma_{13} c_{33} \partial_{x} w_{1}\right]+\frac{h_{\mathrm{e}}^{2}}{8}\left[A_{102} \partial_{x} \partial_{t}^{2} w_{0}+A_{120} \partial_{x} \partial_{y}^{2} w_{0}\right. \\
\left.+A_{300} \partial_{x}^{3} w_{0}+A_{002} \partial_{t}^{2} u_{1}+A_{200} \partial_{x}^{2} u_{1}+A_{020} \partial_{y}^{2} u_{1}+A_{110} \partial_{x} \partial_{y} v_{1}\right]=0 \\
c_{44}\left(v_{1}+\partial_{y} w_{0}\right)+\frac{\alpha h_{\mathrm{e}}}{2}\left[\rho_{\mathrm{p}} \partial_{t}^{2} v_{0}-k_{66} \partial_{x}^{2} v_{0}-\left(k_{22}+\gamma_{23} c_{23}^{\star}\right) \partial_{y}^{2} v_{0}\right. \\
\left.-\left(k_{126}+\gamma_{23} c_{13}^{\star}\right) \partial_{x} \partial_{y} u_{0}-\gamma_{23} c_{33} \partial_{y} w_{1}\right]+\frac{h_{\mathrm{e}}^{2}}{8}\left[B_{012} \partial_{y} \partial_{t}^{2} w_{0}+B_{210} \partial_{x}^{2} \partial_{y} w_{0}\right. \\
\left.+B_{030} \partial_{y}^{3} w_{0}+B_{002} \partial_{t}^{2} v_{1}+B_{200} \partial_{x}^{2} v_{1}+B_{020} \partial_{y}^{2} v_{1}+B_{110} \partial_{x} \partial_{y} u_{1}\right]=0 \\
-\bar{\rho} \partial_{t}^{2} w_{0}+(1+\alpha)\left[c_{55} \partial_{x}^{2} w_{0}+c_{44} \partial_{y}^{2} w_{0}+c_{55} \partial_{x} u_{1}+c_{44} \partial_{y} v_{1}\right] \\
+\frac{\alpha h_{\mathrm{e}}}{2}\left[-\rho_{\mathrm{p}} \hat{\gamma}_{33} \partial_{t}^{2} w_{1}-\left(c_{13}+\alpha \gamma_{13} c_{33}\right) \partial_{x}^{2} w_{1}-\left(c_{23}+\alpha \gamma_{23} c_{33}\right) \partial_{y}^{2} w_{1}\right. \\
+\left(\bar{\rho}-\alpha \gamma_{13}^{\star} \rho_{\mathrm{p}}\right) \partial_{x} \partial_{t}^{2} u_{0}-\left(\bar{c}_{12}+2 \bar{c}_{66}+\alpha \gamma_{23} c_{13}^{\star}\right) \partial_{x} \partial_{y}^{2} u_{0}-\left(\bar{c}_{11}+\alpha \gamma_{13} c_{13}^{\star}\right) \partial_{x}^{3} u_{0} \\
\left.+\left(\bar{\rho}-\alpha \gamma_{23}^{\star} \rho_{\mathrm{p}}\right) \partial_{y} \partial_{t}^{2} v_{0}-\left(\bar{c}_{12}+2 \bar{c}_{66}+\alpha \gamma_{13} c_{23}^{\star}\right) \partial_{x}^{2} \partial_{y} v_{0}-\left(\bar{c}_{22}+\alpha \gamma_{23} c_{23}^{\star}\right) \partial_{y}^{3} v_{0}\right] \\
-\frac{h_{\mathrm{e}}^{2}}{12}\left[C_{004} \partial_{t}^{4} w_{0}+C_{202} \partial_{x}^{2} \partial_{t}^{2} w_{0}+C_{022} \partial_{y}^{2} \partial_{t}^{2} w_{0}+C_{400} \partial_{x}^{4} w_{0}+C_{040} \partial_{y}^{4} w_{0}\right. \\
+C_{220} \partial_{x}^{2} \partial_{y}^{2} w_{0}+C_{102} \partial_{x} \partial_{t}^{2} u_{1}+C_{120} \partial_{x} \partial_{y}^{2} u_{1}+C_{300} \partial_{x}^{3} u_{1}+C_{012} \partial_{y} \partial_{t}^{2} v_{1} \\
\left.+C_{210} \partial_{x}^{2} \partial_{y} v_{1}+C_{030} \partial_{y}^{3} v_{1}\right]=-\frac{\alpha e_{z 3} \rho_{\mathrm{p}}}{2 k_{33}} \partial_{t}^{2} \Delta V-\frac{\alpha^{3} h_{\mathrm{e}}^{2} \epsilon_{z z} e_{z 3} \rho_{\mathrm{p}}^{2}}{24 k_{33} h_{33}} \partial_{t}^{4} \Delta V
\end{gathered}
$$


Here terms quadratic in $h_{\mathrm{e}}$ are retained. The lengthy expressions for the constants $A_{m n p}$, $B_{m n p}$, and $C_{m n p}$ are given in Appendix A. The indices on these constants correspond to the order of the derivatives in $x, y$, and $t$, respectively.

These antisymmetric equations describe essentially bending motion of the plate and it is therefore necessary to include quadratic terms in $h_{\mathrm{e}}$ to account for the bending stiffness of the plate. The linear terms in $h_{\mathrm{e}}$ account for the coupling to the in-plane equations. Below in the numerical results it is shown that these coupling terms are usually of minor importance.

A few words about the orders of $h_{\mathrm{e}}$ used in the derivations are appropriate here. To obtain second order as given here, it is necessary to include six terms in the original expansions of displacements and electric potential, i.e. to terms $O\left(h_{\mathrm{e}}^{5}\right)$. This gives stresses to order $O\left(h_{\mathrm{e}}^{4}\right)$. When eliminating from seven to six equations one cancelation gives that these equations are of order $O\left(h_{\mathrm{e}}^{3}\right)$, but when these six equations are added and subtracted pairwise there is an additional cancelation so that the final equations are of order $O\left(h_{\mathrm{e}}^{2}\right)$. The way the equations are derived and earlier findings [8] strongly suggest that the equations are asymptotically correct.

Finally, it should be pointed out that there are problems with performing eliminations in the system of differential equations. If Gaussian elimination is used in the elimination procedure, new solutions are introduced when eliminating in more than one step, due to multiplication with differential operators. A more systematic method which does not introduce new solutions and performs the elimination in just one step can instead be devised. However, if this method is used repeatedly, new solutions are still introduced. This implies that if fewer than six equations are asked for, the original system of seven equations should be used as the starting point, and not the six equations obtained after one elimination. It must also be noted that the dispersion relation obtained from the six equations and truncated to order $O\left(h_{\mathrm{e}}^{2}\right)$ is different from the dispersion relation obtained from the seven equations and truncated to order $O\left(h_{\mathrm{e}}^{2}\right)$, although the system of six and seven equations are both of order $O\left(h_{\mathrm{e}}^{2}\right)$.

\section{NUMERICAL RESULTS}

In this section numerical examples are given for guided waves along the plate and for a simple 1D actuator case. The plate equations of the previous section are compared with the exact solution with the main goal of investigating the accuracy of the plate equations.

The piezoelectric layer is taken to be PZT-2 (Lead Zirconate Titanate), which is of class $6 \mathrm{~mm}$, and the elastic layer is isotropic steel with density $\rho_{\mathrm{e}}=7870 \mathrm{~kg} / \mathrm{m}^{3}$, Young's modulus $E=210 \mathrm{GPa}$, and Poisson's ratio $\nu=0.3$. PZT-2 is transversely isotropic with material constants (Auld [12]) $\rho_{\mathrm{p}}=7600 \mathrm{~kg} / \mathrm{m}^{3}, k_{11}=k_{22}=135 \mathrm{GPa}, k_{12}=67.0 \mathrm{GPa}, k_{13}=$ $k_{23}=68.1 \mathrm{GPa}, k_{33}=113 \mathrm{GPa}, k_{44}=k_{55}=22.2 \mathrm{GPa}, e_{x 5}=e_{y 4}=9.8 \mathrm{C} / \mathrm{m}^{2}, e_{z 1}=$ $e_{z 2}=-1.9 \mathrm{C} / \mathrm{m}^{2}, e_{z 3}=9.0 \mathrm{C} / \mathrm{m}^{2}, \epsilon_{x x}=\epsilon_{y y}=504 \epsilon_{0}$, and $\epsilon_{z z}=260 \epsilon_{0}$. Here $\epsilon_{0}=$ $8.854 \cdot 10^{-12} \mathrm{C} / \mathrm{Vm}$ is the dielectric permittivity of free space. For a transversely isotropic material $k_{66}=\frac{1}{2}\left(k_{11}-k_{12}\right)$. 
Only time harmonic conditions are considered with the time factor $\mathrm{e}^{-\mathrm{i} \omega t}$. The dimensionless variable $\Omega=\omega h \sqrt{\rho_{\mathrm{e}} / c_{55}}$ is used to measure the frequency. Here $h=h_{\mathrm{e}}+h_{\mathrm{p}}$ is the total thickness of the plate.

Consider first the free propagating modes in the plate. The piezoelectric layer is taken to be short-circuited $\Delta V=0$. This means that the piezoelectric effect has a rather small influence, at least at low frequencies. As PZT-2 is transversely isotropic the direction of propagation in the $x y$ plane is immaterial. The wave number in the direction of propagation is $k$. The dispersion relation is plotted as frequency $\Omega$ versus dimensionless wave number $k h$. The results of the plate equations (given by full-drawn lines in the figures) are compared with the exact solution (given by dashed lines).

Figure 2 shows the dispersion curves when the piezoelectric layer is one fourth of the total thickness $(\alpha=1 / 3)$. Three pairs of curves are seen. The almost straight line from the

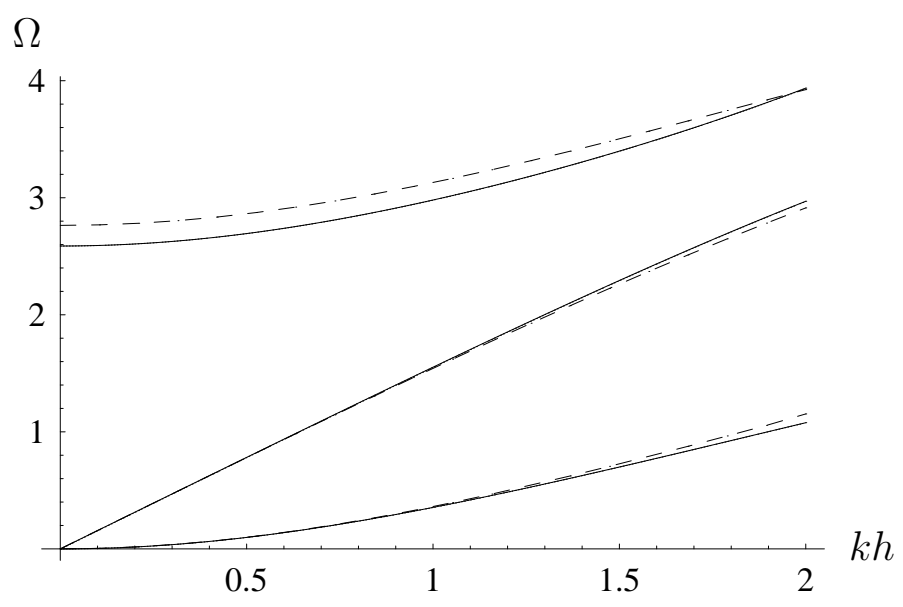

FiguRE 2. Dispersion curves for a thin piezoelectric layer $(\alpha=1 / 3)$, comparing plate equation (full-drawn) and exact (dashed) solutions.

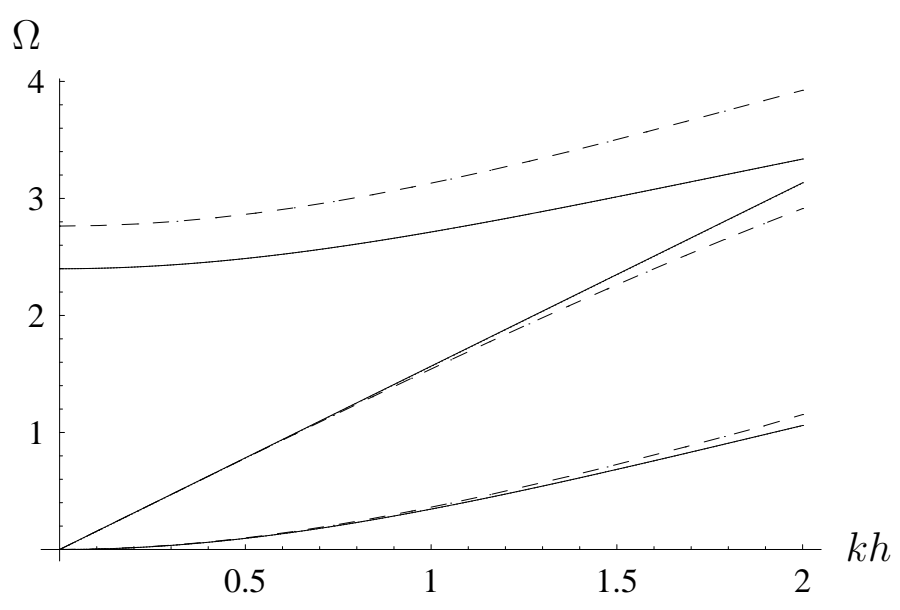

FIGURE 3. Dispersion curves for a thin piezoelectric layer $(\alpha=1 / 3)$ with neglected coupling between in-plane and bending motions, comparing plate equation (full-drawn) and exact (dashed) solutions. 
origin is the first (almost) in-plane mode which is dispersion-free at low frequencies. The other two curves are (almost) bending modes, with the first one behaving as a parabola at low frequencies. The second bending mode and its cut-off are not very accurately predicted by the plate equations, but the two lowest modes are very well approximated at low frequencies.

Figure 3 shows the same dispersion curves but with all coupling between the in-plane and bending motions neglected, i.e. with the terms linear i $h_{\mathrm{e}}$ in Eqs. (4.1)-(4.6) suppressed. Although it is seen that the solution is not as accurate, the difference between Figs. 2 and 3 is very minor at low frequencies and this shows that the coupling between the in-plane and bending motion is small.

Figures 4 and 5 are similar to Figs. 2 and 3 but with a piezoelectric layer that has the same thickness as the steel $(\alpha=1)$. The results are almost as good as previously, although the decoupling approximation is somewhat less accurate than in the previous case. It is

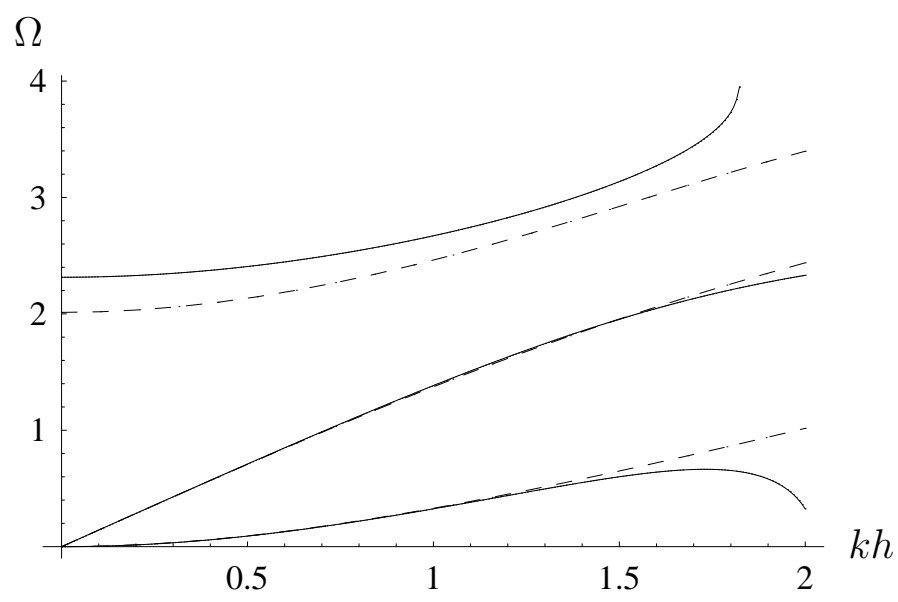

FiguRE 4. Dispersion curves for a thick piezoelectric layer $(\alpha=1)$, comparing plate equation (full-drawn) and exact (dashed) solutions.

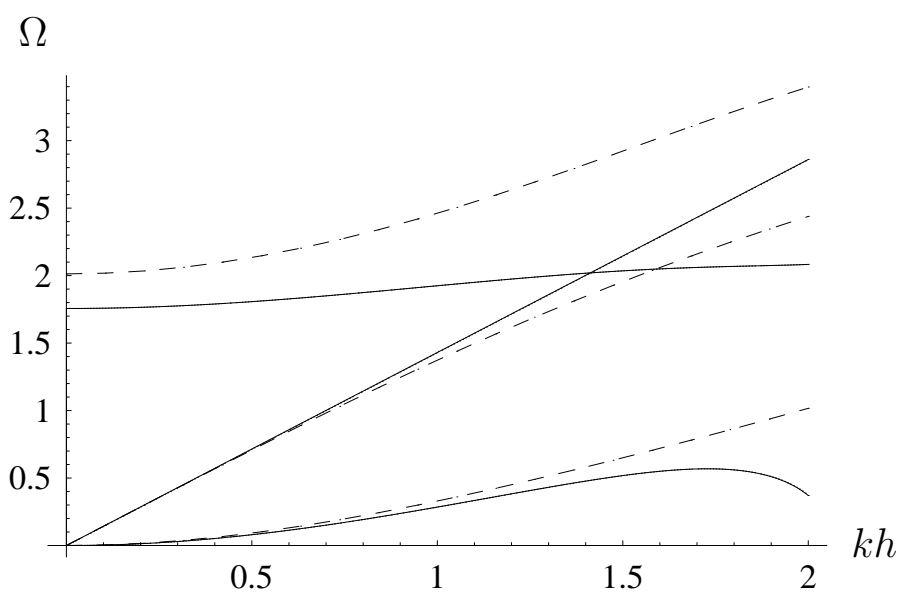

FIGURE 5. Dispersion curves for a thick piezoelectric layer $(\alpha=1)$ with neglected coupling between in-plane and bending motions, comparing plate equation (full-drawn) and exact (dashed) solutions. 
seen that great errors are obtained in Fig. 4 as $k h$ is around 2. The first (approximate) bending wave has a complex branch on its dispersion curve when $k h$ is a little greater than 2 , and for the second bending wave this happens already for $k h$ a little less than 2 , but this curve is not a very good approximation anyway. Another point worth noting is that one of the bending curves is crossing the in-plane curve in Fig. 5; this is of course a behaviour that is not allowed if coupling is taken into account.

To further investigate the accuracy of the plate equations, also the displacement components and the electric potential in the modes are plotted. It must then be noted that it is straightforward to obtain the displacement components in the elastic layer from the six plate equations of the previous section, but the displacement components and the electric potential in the piezoelectric layer is not easily obtained from the solution of these six equations, the problem being that there is no direct way to calculate the expansion function $\Phi_{1}$. This problem can be circumvented by using the seven equations to calculate the fields

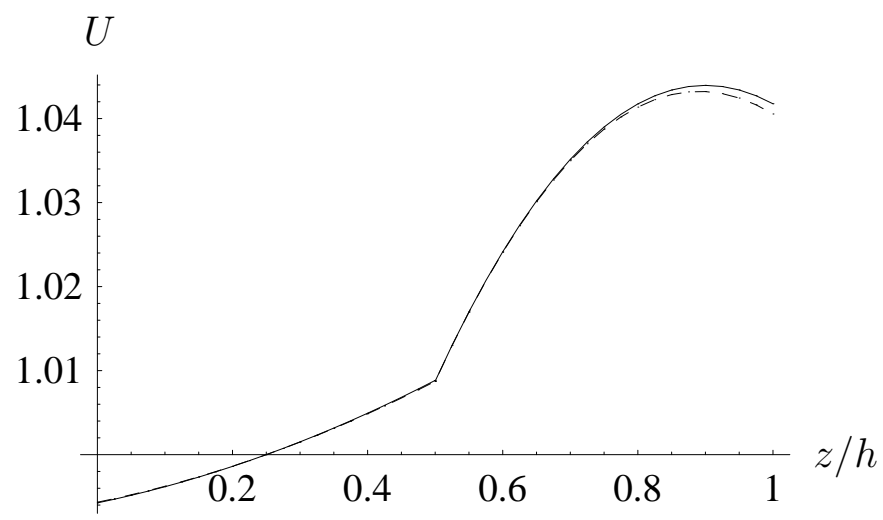

FiguRE 6. The horizontal displacement component $U$ through the thickness of the plate for the first in-plane mode for a thick piezoelectric layer $(\alpha=1)$ and the frequency $\Omega=0.5$, comparing plate equation (full-drawn) and exact (dashed) solutions.

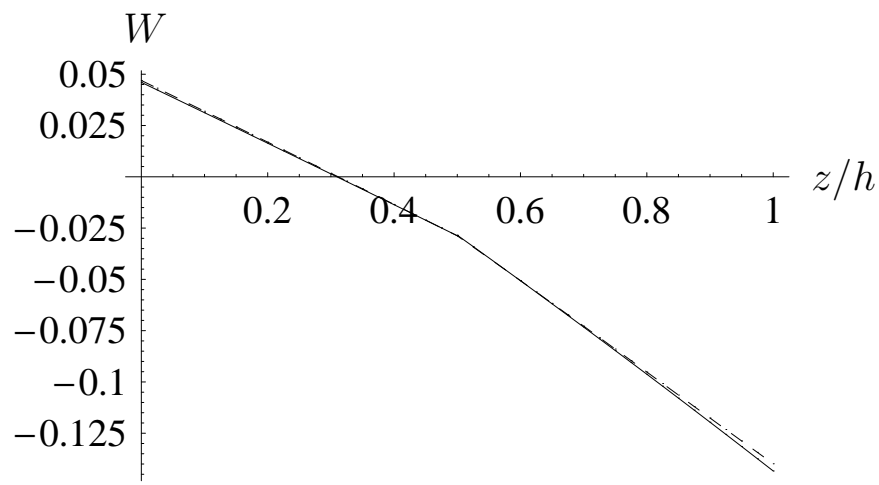

FIGURE 7 . The vertical displacement component $W$ through the thickness of the plate for the first in-plane mode for a thick piezoelectric layer $(\alpha=1)$ and the frequency $\Omega=0.5$, comparing plate equation (full-drawn) and exact (dashed) solutions. 


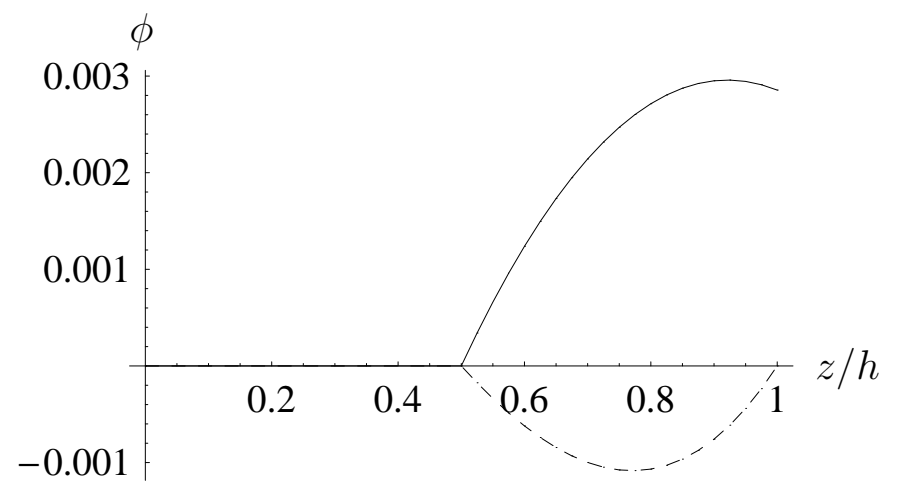

FIGURE 8. The electric potential $\phi$ through the thickness of the plate for the first in-plane mode for a thick piezoelectric layer $(\alpha=1)$ and the frequency $\Omega=0.5$, comparing plate equation (full-drawn) and exact (dashed) solutions.

in the piezoelectric layer as described in the previous section and this is thus done here. However, the displacement components in the elastic layer are still computed from the six equations. The reason for this is that the six equations are probably the most useful ones and therefore the accuracy of these are of most interest. The difference between using six or seven equations in the elastic layer is very minor though.

Only the case $\alpha=1$ is considered, i.e. the piezoelectric and elastic layers are of equal thickness. The frequency is chosen to be $\Omega=0.5$ and it can not be much higher for the existence of the first approximate bending mode, corresponding to the wave number $k h=1.307$ at this frequency, see Fig. 4. For the plots a $z$ axis is chosen so that $z=0$ corresponds to the lower surface and $z=h$ to the upper. The first in-plane and bending modes are plotted; the in-plane one is normalized so that the horizontal displacement $U=1$ in the middle of the elastic layer at $z / h=0.25$ and the bending one is normalized so that the vertical displacement $W=1$ in the middle of the elastic layer. The dimensionless electric potential is defined as $\phi=\Phi \epsilon_{x x} /\left(h e_{x 5}\right)$.

Figures 6-8 show $U, W$, and $\phi$ for the in-plane mode. For the displacement components it is seen that the approximate and exact solutions agree very well. As expected the displacement is continuous but its derivative is discontinuous at the interface between the piezoelectric and elastic layer at $z / h=0.5$. For the electric potential the approximate and exact solutions do not agree at all and it is also seen that the approximate solution does not fulfill the boundary condition $\phi=0$ at the upper surface. As is seen from the scale, however, the electric potential in this mode is very small so the error is still small, although it is a little puzzling that not even the sign is correct.

Figures 9-11 similarly show the displacements and electric potential for the first bending mode. Here the discrepancies between the exact and approximate solutions are a little larger. But the scales should be observed; the errors relative the maximum are only a few percent for the displacements. For this mode the potential is much larger (about a factor 50) than for the in-plane mode, but both the absolute and relative errors between the exact and approximate solutions are fairly large. It is also noted that displacements are discontinuous at the interface for this case although the jumps are quite small. 


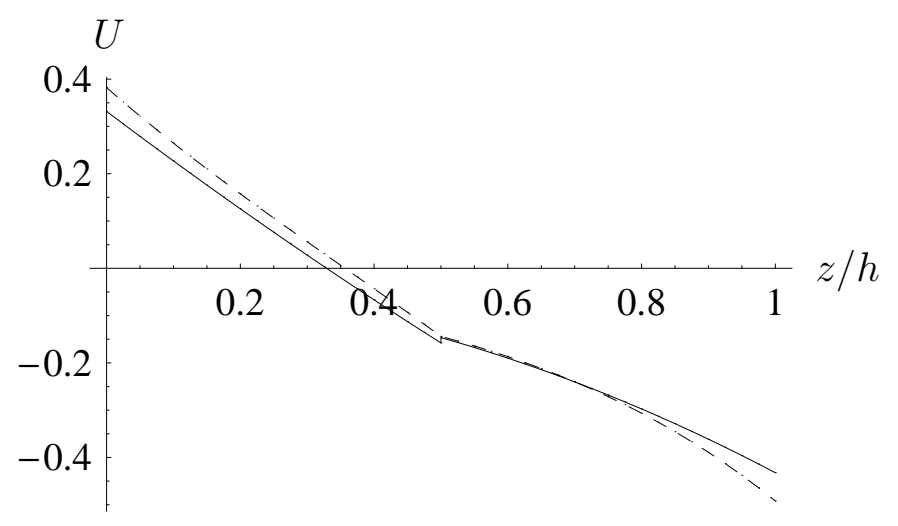

FiguRE 9. The horizontal displacement component $U$ through the thickness of the plate for the first bending mode for a thick piezoelectric layer $(\alpha=1)$ and the frequency $\Omega=0.5$, comparing plate equation (full-drawn) and exact (dashed) solutions.

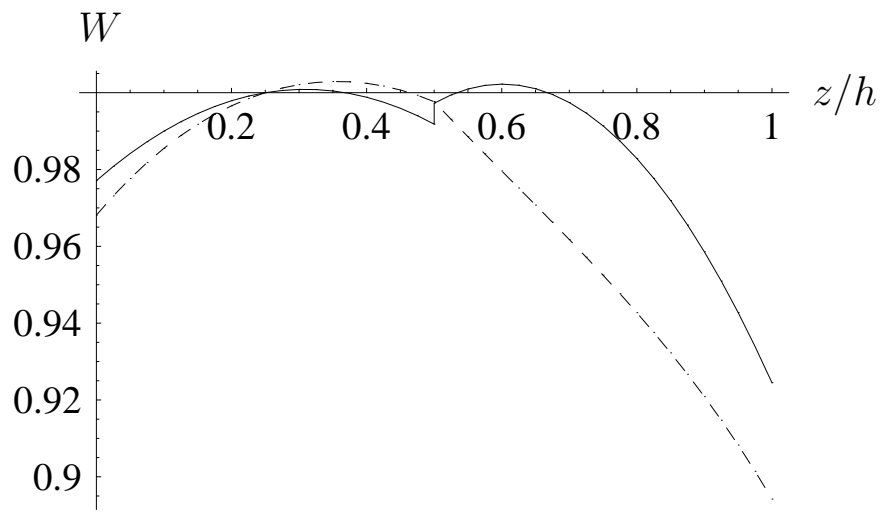

Figure 10. The vertical displacement component $W$ through the thickness of the plate for the first bending mode for a thick piezoelectric layer $(\alpha=1)$ and the frequency $\Omega=0.5$, comparing plate equation (full-drawn) and exact (dashed) solutions.

As a final example, a simple actuator example is considered. As both layers are infinite this becomes a 1D problem with a given jump in potential (with a fixed frequency as above) leading to a vertical displacement. At zero frequency the exact solution for the amplitude in the middle of the elastic layer is

$$
w_{00}=\frac{\alpha \rho_{\mathrm{p}} e_{z 3} \Delta V}{2 k_{33}\left(\rho_{\mathrm{e}}+\alpha \rho_{\mathrm{p}}\right)} .
$$

This is also the solution that is obtained at zero frequency (or zero plate thickness) from Eqs. (4.3) and (4.6), again showing the asymptotic correctness of the plate equations. Keeping all orders in the thickness in Eqs. (4.3) and (4.6) leads to the result in Fig. 12 , where the displacement is plotted as a function of frequency. The displacement is normalized with the value at zero frequency as given by Eq. (5.1). The elastic and piezoelectric layers are of the same thickness, i.e. $\alpha=1$. The exact solution is plotted 


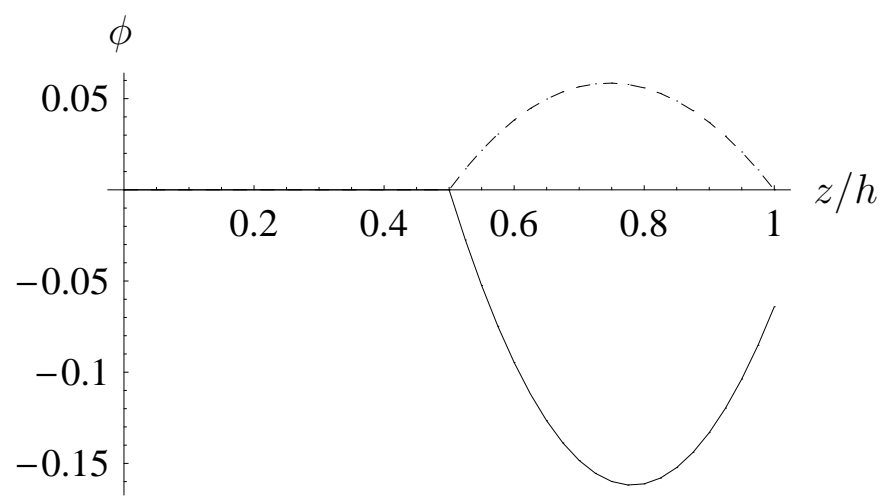

FiguRE 11. The electric potential $\phi$ through the thickness of the plate for the first bending mode for a thick piezoelectric layer $(\alpha=1)$ and the frequency $\Omega=0.5$, comparing plate equation (full-drawn) and exact (dashed) solutions.

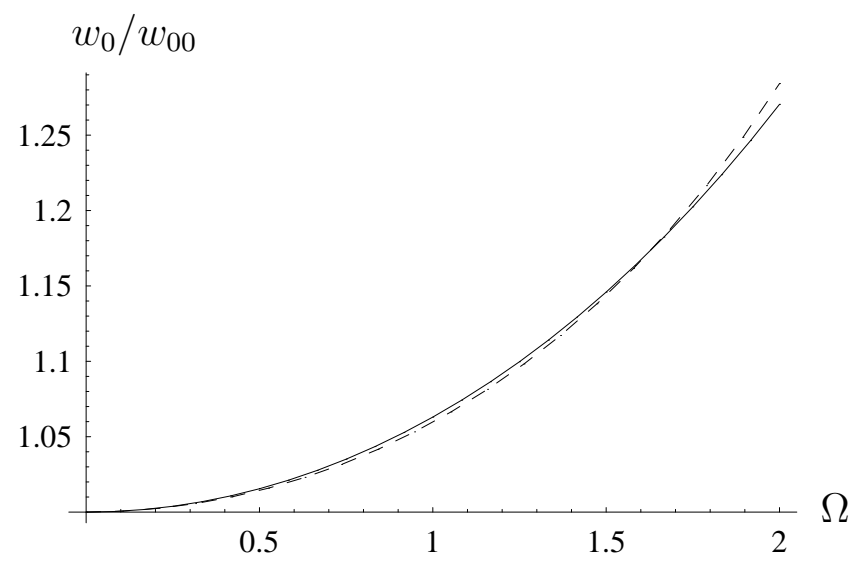

FIGURE 12. The normalized vertical displacement as a function of frequency for a $1 \mathrm{D}$ actuator case.

with a dashed line and the approximate one with a full-drawn line. It is seen that the agreement is very good, in fact better than for the dispersion relations.

\section{Concluding Remarks}

The derivation of plate equations for a two-layered plate with one elastic and one piezoelectric layer is considered. The equations become very complicated, but there are good reasons to believe that they are asymptotically correct. For sensor and actuator applications it may very well be possible to simplify the equations considerably, e.g. by dropping terms that contribute bending rigidity due to the piezoelectric layer. If the piezoelectric layer is thin compared to the elastic one it should be a good approximation to drop all terms quadratic or higher in $\alpha$.

Only an infinite plate is considered here, but there are of course good reasons to go further and investigate finite piezoelectric patches on a larger plate. This can be done either 
by using the present equations or by using equivalent boundary conditions [9, 11] and comparing with exact calculations for simple cases.

The present approach is of course also applicable to purely elastic, layered plates, and for this case the problems with the expansion function of electric potential $\Phi_{1}$ do not arise. Instead it should be possible to recursively eliminate the expansion functions for one layer at a time, starting at the two surfaces and ending with six equations for the middle layer. For a symmetric plate this could be done for the symmetric and antisymmetric parts separately.

\section{ACKNOWLEDGEMENT}

The present work is sponsored by the Swedish Research Council and this is gratefully acknowledged.

\section{ApPendix A}

This Appendix lists all the constants introduced in the plate equations in Section 4. The notation partly reflects the nature of the constants; thus all constants with the same letter but different indices have the same dimension and the indices more or less reflect the indices of the underlying material constants. In the elastic layer the first three constants introduced for the piezoelectric layer in Eq. (3.9) have a counterpart:

$$
\begin{aligned}
& c_{126}=c_{12}+c_{66}, \\
& c_{135}=c_{13}+c_{55}, \\
& c_{234}=c_{23}+c_{44} .
\end{aligned}
$$

In the symmetric plate equations (4.1)-(4.3) some further constants are introduced. Essentially the mean through the thickness enters in some places and they are defined as follows

$$
\begin{aligned}
& \bar{\rho}=\rho_{\mathrm{e}}+\alpha \rho_{\mathrm{p}}, \\
& \bar{c}_{i j}=c_{i j}+\alpha k_{i j}, \\
& \bar{c}_{i j k}=c_{i j k}+\alpha k_{i j k} .
\end{aligned}
$$

Also the difference is sometimes used

$$
c_{i j}^{\star}=c_{i j}-k_{i j} .
$$

Two new stiffnesses are introduced

$$
\begin{aligned}
& k_{113}=k_{11}-k_{13}^{2} / k_{33}, \\
& k_{223}=k_{22}-k_{23}^{2} / k_{33},
\end{aligned}
$$


and some dimensionless constants (no summation convention)

$$
\begin{aligned}
\gamma_{i j} & =k_{i j} / k_{j j}, \\
\gamma_{i j}^{\star} & =c_{i j}^{\star} / k_{j j}, \\
\hat{\gamma}_{i j} & =\left(k_{i j}+\alpha c_{i j}\right) / k_{j j}, \\
\beta_{i j} & =c_{i j} / c_{j j}, \\
\kappa_{i i} & =c_{i i} / k_{i i} .
\end{aligned}
$$

Here the last two definitions are needed below.

In the antisymmetric plate equations (4.4)-(4.6) the coefficients in front of the terms quadratic in $h_{\mathrm{e}}$ are given by quite lengthy expressions

$$
\begin{aligned}
A_{102}= & -\beta_{13} \rho_{\mathrm{e}}-2 \alpha\left(\gamma_{13} \rho_{\mathrm{e}}-\alpha \gamma_{55}^{\star} \rho_{\mathrm{p}}\right)+\frac{2 \alpha^{2} \rho_{\mathrm{p}}}{h_{33}}\left[h_{55}-h_{135}-e_{z 1} e_{z 3}+e_{z 3}^{2}\left(\gamma_{13}+\frac{2 c_{55}}{3 k_{33}}\right)\right], \\
A_{120}= & -\beta_{13} c_{44}+2 \alpha\left[\alpha\left(\gamma_{44}^{\star} k_{126}+\gamma_{55}^{\star} k_{66}\right)-\gamma_{13} c_{44}\right]+\frac{2 \alpha^{2}}{h_{33}}\left[-k_{55}\left(\gamma_{44}^{\star} h_{234}+h_{44}\right)\right. \\
& \left.+h_{135}\left(k_{44}+\gamma_{44}^{\star} k_{234}\right)-g_{135}\left(\gamma_{44}^{\star} e_{z 2}+\kappa_{44} e_{y 4}\right)+\left(g_{43}+\gamma_{44}^{\star} g_{234}\right)\left(e_{51}-\gamma_{13} e_{z 3}\right)\right] \\
& +\frac{4 \alpha^{2} c_{55}}{3 k_{44} h_{33}}\left[k_{44}\left(h_{34}-g_{43} e_{z 3} / k_{33}\right)+g_{234}\left(-e_{42}+e_{z 3} k_{234} / k_{33}\right)\right], \\
A_{300}= & \beta_{13} c_{55}+2 \alpha\left[\gamma_{13} c_{55}-\alpha \gamma_{55}^{\star} k_{11}\right]+\frac{4 \alpha^{2}}{3 h_{33}}\left[c_{55}\left(h_{35}-g_{53} e_{z 3} / k_{33}\right)\right. \\
& \left.+\kappa_{55} g_{135}\left(e_{z 3} k_{135} / k_{33}-e_{51}\right)\right]+\frac{2 \alpha^{2}}{h_{33}}\left[k_{55}\left(h_{135}-h_{55}\right)\right. \\
& \left.+\gamma_{55}^{\star} k_{13} h_{135}+g_{53}\left(e_{51}-\gamma_{13} e_{z 3}\right)-g_{135}\left(\gamma_{13} \gamma_{55}^{\star} e_{z 3}+e_{x 5}\right)\right], \\
A_{020}= & -c_{66}-2 \alpha \hat{\gamma}_{55} k_{66}+\frac{4 \alpha^{2} c_{55} h_{34}}{3 h_{33}}+\frac{4 \alpha^{2} c_{55}}{3 k_{33} k_{44} h_{33}}\left[g_{234}\left(k_{234} e_{z 3}-k_{33} e_{42}\right)-k_{44} g_{43} e_{z 3}\right], \\
A_{200}= & -c_{11}+\beta_{13} c_{135}+2 \alpha\left[\gamma_{13}\left(k_{13}+c_{55}\right)-k_{11}\right]+\frac{2 \alpha^{2} \kappa_{55}}{h_{33}}\left(k_{13} h_{135}-\gamma_{13} g_{135} e_{z 3}\right) \\
& +\frac{4 \alpha^{2} c_{55}}{3 h_{33}}\left[h_{35}+\frac{g_{135}}{k_{33} k_{55}}\left(k_{135} e_{z 3}-k_{33} e_{z 1}\right)-g_{53} e_{z 3} / k_{33}\right]-2 \alpha^{2} \kappa_{55} k_{11}, \\
A_{002}= & \bar{\rho}+\alpha \rho_{\mathrm{p}}\left(1+2 \alpha \kappa_{55}\right)+\frac{4 \alpha^{2} c_{55} e_{z 3}^{2} \rho_{\mathrm{p}}}{3 k_{33} h_{33}},
\end{aligned}
$$




$$
\begin{aligned}
& A_{110}=-c_{126}+\beta_{13} c_{234}-2 \alpha \hat{\gamma}_{44} k_{126}-2 \alpha \gamma_{13}\left(c_{44}+k_{23}\right) \\
& +\frac{2 \alpha^{2} \kappa_{44}}{h_{33}}\left[k_{234} h_{135}-k_{55} h_{234}-g_{135} e_{42}+g_{234}\left(e_{51}-\gamma_{13} e_{z 3}\right)\right] \\
& B_{012}=-\beta_{23} \rho_{\mathrm{e}}-2 \alpha\left(\gamma_{23} \rho_{\mathrm{e}}-\alpha \gamma_{44}^{\star} \rho_{\mathrm{p}}\right)+\frac{2 \alpha^{2} \rho_{\mathrm{p}}}{h_{33}}\left[h_{44}-h_{234}-e_{z 2} e_{z 3}+e_{z 3}^{2}\left(\gamma_{23}+\frac{2 c_{44}}{3 k_{33}}\right)\right], \\
& B_{210}=-\beta_{23} c_{55}+2 \alpha\left[\alpha\left(\gamma_{55}^{\star} k_{126}+\gamma_{44}^{\star} k_{66}\right)-\gamma_{23} c_{55}\right]+\frac{2 \alpha^{2}}{h_{33}}\left[-k_{44}\left(\gamma_{55}^{\star} h_{135}+h_{55}\right)\right. \\
& \left.+h_{234}\left(k_{55}+\gamma_{55}^{\star} k_{135}\right)-g_{234}\left(\gamma_{55}^{\star} e_{z 1}+\kappa_{55} e_{x 5}\right)+\left(g_{53}+\gamma_{55}^{\star} g_{135}\right)\left(e_{42}-\gamma_{23} e_{z 3}\right)\right] \\
& +\frac{4 \alpha^{2} c_{44}}{3 k_{55} h_{33}}\left[k_{55}\left(h_{35}-g_{53} e_{z 3} / k_{33}\right)+g_{135}\left(-e_{51}+e_{z 3} k_{135} / k_{33}\right)\right] \text {, } \\
& B_{030}=\beta_{23} c_{44}+2 \alpha\left[\gamma_{23} c_{44}-\alpha \gamma_{44}^{\star} k_{22}\right]+\frac{4 \alpha^{2}}{3 h_{33}}\left[c_{44}\left(h_{34}-g_{43} e_{z 3} / k_{33}\right)\right. \\
& \left.+\kappa_{44} g_{234}\left(e_{z 3} k_{234} / k_{33}-e_{42}\right)\right]+\frac{2 \alpha^{2}}{h_{33}}\left[k_{44}\left(h_{234}-h_{44}\right)\right. \\
& \left.+\gamma_{44}^{\star} k_{23} h_{234}+g_{43}\left(e_{42}-\gamma_{23} e_{z 3}\right)-g_{234}\left(\gamma_{23} \gamma_{44}^{\star} e_{z 3}+e_{y 4}\right)\right] \\
& B_{002}=\bar{\rho}+\alpha \rho_{\mathrm{p}}\left(1+2 \alpha \kappa_{44}\right)+\frac{4 \alpha^{2} c_{44} e_{z 3}^{2} \rho_{\mathrm{p}}}{3 k_{33} h_{33}}, \\
& B_{020}=-c_{22}+\beta_{23} c_{234}+2 \alpha\left[\gamma_{23}\left(k_{23}+c_{44}\right)-k_{22}\right]+\frac{2 \alpha^{2} \kappa_{44}}{h_{33}}\left(k_{23} h_{234}-\gamma_{23} g_{234} e_{z 3}\right) \\
& +\frac{4 \alpha^{2} c_{44}}{3 h_{33}}\left[h_{34}+\frac{g_{234}}{k_{33} k_{44}}\left(k_{234} e_{z 3}-k_{33} e_{z 2}\right)-g_{43} e_{z 3} / k_{33}\right]-2 \alpha^{2} \kappa_{44} k_{22}, \\
& B_{200}=-c_{66}-2 \alpha \hat{\gamma}_{44} k_{66}+\frac{4 \alpha^{2} c_{44} h_{35}}{3 h_{33}}+\frac{4 \alpha^{2} c_{44}}{3 k_{33} k_{55} h_{33}}\left[g_{135}\left(k_{135} e_{z 3}-k_{33} e_{51}\right)-k_{55} g_{53} e_{z 3}\right], \\
& B_{110}=-c_{126}+\beta_{23} c_{135}-2 \alpha \hat{\gamma}_{55} k_{126}-2 \alpha \gamma_{23}\left(c_{55}+k_{13}\right) \\
& +\frac{2 \alpha^{2} \kappa_{55}}{h_{33}}\left[k_{135} h_{234}-k_{44} h_{135}-g_{234} e_{51}+g_{135}\left(e_{42}-\gamma_{23} e_{z 3}\right)\right] \text {, } \\
& C_{004}=\frac{\rho_{\mathrm{e}}}{2 c_{33}}\left[\rho_{\mathrm{e}}+3 \alpha \rho_{\mathrm{p}}\left(1+2 \alpha \kappa_{33}\right)\right]+\frac{\alpha^{2} \rho_{\mathrm{p}}}{k_{33} h_{33}}\left[2 \alpha k_{33} \epsilon_{z z} \rho_{\mathrm{p}}+e_{z 3}^{2}\left(2 \rho_{\mathrm{e}}-\alpha \rho_{\mathrm{p}}\right)\right],
\end{aligned}
$$




$$
\begin{aligned}
C_{202}= & \frac{1}{2 c_{33}}\left[c_{13} \rho_{\mathrm{e}}(1+3 \alpha)-c_{55}\left(\rho_{\mathrm{e}}+3 \alpha \rho_{\mathrm{p}}\right)\right] \\
& -2 \alpha^{3} \kappa_{55} \rho_{\mathrm{p}}+\alpha^{2}\left[3 \gamma_{13} \rho_{\mathrm{e}}+\rho_{\mathrm{p}}\left(2 \alpha-3 c_{55} / k_{33}\right)\right] \\
& +\frac{\alpha^{2}}{h_{33}}\left[2 \bar{\rho}\left(h_{35}+g_{135} e_{51} / k_{55}\right)-e_{z 3} \rho_{\mathrm{p}}\left(3 \alpha\left(g_{135} / k_{33}+\gamma_{13} e_{z 3}\right)\right.\right. \\
& \left.+\frac{\kappa_{55}}{k_{33}}\left(-3 \alpha g_{135}+2 k_{55} e_{z 3}(1+\alpha)\right)\right)-\frac{g_{53} e_{z 3}}{k_{33}}\left(2 \rho_{\mathrm{e}}-\alpha \rho_{\mathrm{p}}\right) \\
& \left.+\frac{2 k_{135} g_{135} e_{z 3} \bar{\rho}}{k_{33} k_{55}}-\alpha \rho_{\mathrm{p}}\left(2 \kappa_{55} h_{135}+4\left(h_{55}-h_{135}\right)-3 e_{z 1} e_{z 3}\right)\right]
\end{aligned}
$$

$$
\begin{aligned}
C_{022}= & \frac{1}{2 c_{33}}\left[c_{23} \rho_{\mathrm{e}}(1+3 \alpha)-c_{44}\left(\rho_{\mathrm{e}}+3 \alpha \rho_{\mathrm{p}}\right)\right] \\
& -2 \alpha^{3} \kappa_{44} \rho_{\mathrm{p}}+\alpha^{2}\left[3 \gamma_{23} \rho_{\mathrm{e}}+\rho_{\mathrm{p}}\left(2 \alpha-3 c_{44} / k_{33}\right)\right] \\
& +\frac{\alpha^{2}}{h_{33}}\left[2 \bar{\rho}\left(h_{34}+g_{234} e_{42} / k_{44}\right)-e_{z 3} \rho_{\mathrm{p}}\left(3 \alpha\left(g_{234} / k_{33}+\gamma_{23} e_{z 3}\right)\right.\right. \\
& \left.+\frac{\kappa_{44}}{k_{33}}\left(-3 \alpha g_{234}+2 k_{44} e_{z 3}(1+\alpha)\right)\right)-\frac{g_{43} e_{z 3}}{k_{33}}\left(2 \rho_{\mathrm{e}}-\alpha \rho_{\mathrm{p}}\right) \\
& \left.+\frac{2 k_{234} g_{234} e_{z 3} \bar{\rho}}{k_{33} k_{44}}-\alpha \rho_{\mathrm{p}}\left(2 \kappa_{44} h_{234}+4\left(h_{44}-h_{234}\right)-3 e_{z 2} e_{z 3}\right)\right]
\end{aligned}
$$

$$
\begin{aligned}
C_{400}= & -\frac{1}{2} \beta_{13} c_{55}(1+3 \alpha)-\alpha^{2}\left(3 \gamma_{13} c_{55}-2 \alpha \gamma_{55}^{\star} k_{11}\right)-\frac{2 \alpha^{2}(1+\alpha) c_{55} h_{35}}{h_{33}} \\
& +\frac{\alpha^{3}}{h_{33}}\left[2 k_{55} h_{55}+2 h_{135}\left(k_{13}-k_{55}\right)+g_{53}\left(3 \gamma_{13} e_{z 3}-3 e_{z 1}-2 e_{x 5}\right)\right. \\
& \left.+g_{135}\left(2 e_{x 5}+e_{z 1}-3 \gamma_{13} e_{z 3}\right)\right]+\frac{\alpha^{2} \kappa_{55} e_{z 3}}{k_{33} h_{33}}\left[2(1+\alpha) k_{55} g_{53}+g_{135}\left(3 \alpha k_{13}\right.\right. \\
& \left.\left.-2(1+\alpha) k_{135}\right)\right]+\frac{\alpha^{2} \kappa_{55}}{h_{33}}\left[g_{135}\left(2(1+\alpha) e_{51}-\alpha e_{z 1}\right)-2 \alpha k_{13} h_{135}\right]
\end{aligned}
$$

$$
\begin{aligned}
C_{040}= & -\frac{1}{2} \beta_{23} c_{44}(1+3 \alpha)-\alpha^{2}\left(3 \gamma_{23} c_{44}-2 \alpha \gamma_{44}^{\star} k_{22}\right)-\frac{2 \alpha^{2}(1+\alpha) c_{44} h_{34}}{h_{33}} \\
& +\frac{\alpha^{3}}{h_{33}}\left[2 k_{44} h_{44}+2 h_{234}\left(k_{23}-k_{44}\right)+g_{43}\left(3 \gamma_{23} e_{z 3}-3 e_{z 2}-2 e_{y 4}\right)\right. \\
& \left.+g_{234}\left(2 e_{y 4}+e_{z 2}-3 \gamma_{23} e_{z 3}\right)\right]+\frac{\alpha^{2} \kappa_{44} e_{z 3}}{k_{33} h_{33}}\left[2(1+\alpha) k_{44} g_{43}+g_{234}\left(3 \alpha k_{23}\right.\right. \\
& \left.\left.-2(1+\alpha) k_{234}\right)\right]+\frac{\alpha^{2} \kappa_{44}}{h_{33}}\left[g_{234}\left(2(1+\alpha) e_{42}-\alpha e_{z 2}\right)-2 \alpha k_{23} h_{234}\right]
\end{aligned}
$$




$$
\begin{aligned}
& C_{220}=-\frac{1}{2}(1+3 \alpha)\left(\beta_{13} c_{44}+\beta_{23} c_{55}\right) \\
& +2 \alpha^{3}\left(k_{12}+2 k_{66}\right)\left(\kappa_{44}+\kappa_{55}-2\right)-3 \alpha^{2}\left(\gamma_{13} c_{44}+\gamma_{23} c_{55}\right) \\
& +\frac{\alpha^{2}(1+\alpha)}{k_{33} h_{33}}\left[2 e_{z 3}\left(c_{44} g_{53}+c_{55} g_{43}\right)-2 k_{33}\left(c_{44} h_{35}+c_{55} h_{34}\right)\right. \\
& \left.+\frac{2 c_{44} g_{135}}{k_{55}}\left(k_{33} e_{51}-k_{135} e_{z 3}\right)+\frac{2 c_{55} g_{234}}{k_{44}}\left(k_{33} e_{42}-k_{234} e_{z 3}\right)\right] \\
& +\frac{\alpha^{3}}{h_{33}}\left[\kappa_{44}\left(k_{33} e_{42} e_{z 1}+k_{234} e_{z 3}\left(3 \gamma_{13} e_{z 3}-e_{z 1}\right)-k_{13}\left(e_{z 3} e_{42}+2 k_{234} \epsilon_{z z}\right)\right)\right. \\
& +\kappa_{55}\left(k_{33} e_{51} e_{z 2}+k_{135} e_{z 3}\left(3 \gamma_{23} e_{z 3}-e_{z 2}\right)-k_{23}\left(e_{z 3} e_{51}+2 k_{135} \epsilon_{z z}\right)\right) \\
& \left.+2\left(e_{z 3}\left(k_{23} e_{z 1}+k_{13} e_{z 2}\right)-k_{33} e_{z 1} e_{z 2}-3 \gamma_{13} k_{23} e_{z 3}^{2}+2 k_{13} k_{23} \epsilon_{z z}\right)\right] \text {, } \\
& C_{102}=-\frac{c_{135}}{2 c_{33}}\left(\rho_{\mathrm{e}}+3 \alpha \rho_{\mathrm{p}}\right)-2 \alpha^{3} \kappa_{55} \rho_{\mathrm{p}}-3 \alpha^{2} \rho_{\mathrm{p}}\left(1+\gamma_{13}+c_{55} / k_{33}\right)-\frac{1}{2} \rho_{\mathrm{e}}(1+3 \alpha) \\
& +\frac{\alpha^{2} \kappa_{55} \rho_{\mathrm{p}}}{k_{33} h_{33}}\left(\alpha\left(3 g_{135} e_{z 3}-2 k_{33} h_{135}\right)-2(1+\alpha) k_{55} e_{z 3}^{2}\right), \\
& C_{120}=\frac{1}{2}(1+3 \alpha)\left(c_{12}+2 c_{66}-\beta_{23} c_{135}\right)+\alpha^{2}\left(2 \alpha \kappa_{55}+3\right)\left(k_{12}+2 k_{66}\right) \\
& -3 \alpha^{2} \gamma_{23}\left(c_{55}+k_{13}\right)+\frac{\alpha^{2}}{h_{33}}\left[\alpha \kappa _ { 5 5 } \left(2 k_{44} h_{135}-2 k_{135} h_{234}+2 g_{234} e_{51}\right.\right. \\
& \left.-2 g_{135} e_{y 4}-3 g_{135} e_{z 2}+3 \gamma_{23} g_{135} e_{z 3}\right)+2(1+\alpha) c_{55}\left(-h_{34}+g_{43} e_{z 3} / k_{33}\right. \\
& \left.\left.+\frac{g_{234}}{k_{33} k_{44}}\left(k_{33} e_{42}-k_{234} e_{z 3}\right)\right)\right] \text {, } \\
& \begin{aligned}
C_{300}= & \frac{1}{2}(1+3 \alpha)\left(c_{11}-\beta_{13} c_{135}\right)+\alpha^{2}\left[2 \alpha \kappa_{55} k_{11}-3\left(\gamma_{13}\left(c_{55}-k_{13}\right)-k_{11}\right)\right] \\
& -\frac{\alpha^{2} \kappa_{55}}{h_{33}}\left[2 k_{55} h_{35}(1+\alpha)+\frac{e_{z 3}}{k_{33}}\left(-2 g_{53} k_{55}(1+\alpha)+g_{135}\left(2 k_{135}(1+\alpha)-3 \alpha k_{13}\right)\right)\right. \\
& \left.+2 \alpha k_{13} h_{135}+g_{135}\left(\alpha e_{z 1}-2 e_{51}(1+\alpha)\right)\right],
\end{aligned} \\
& C_{012}=-\frac{c_{234}}{2 c_{33}}\left(\rho_{\mathrm{e}}+3 \alpha \rho_{\mathrm{p}}\right)-2 \alpha^{3} \kappa_{44} \rho_{\mathrm{p}}-3 \alpha^{2} \rho_{\mathrm{p}}\left(1+\gamma_{23}+c_{44} / k_{33}\right)-\frac{1}{2} \rho_{\mathrm{e}}(1+3 \alpha) \\
& +\frac{\alpha^{2} \kappa_{44} \rho_{\mathrm{p}}}{k_{33} h_{33}}\left(\alpha\left(3 g_{234} e_{z 3}-2 k_{33} h_{234}\right)-2(1+\alpha) k_{44} e_{z 3}^{2}\right),
\end{aligned}
$$




$$
\begin{aligned}
C_{210}= & \frac{1}{2}(1+3 \alpha)\left(c_{12}+2 c_{66}-\beta_{13} c_{234}\right)+\alpha^{2}\left(2 \alpha \kappa_{44}+3\right)\left(k_{12}+2 k_{66}\right) \\
& -3 \alpha^{2} \gamma_{13}\left(c_{44}+k_{23}\right)+\frac{\alpha^{2}}{h_{33}}\left[\alpha \kappa _ { 4 4 } \left(2 k_{55} h_{234}-2 k_{234} h_{135}+2 g_{135} e_{42}\right.\right. \\
& \left.-2 g_{234} e_{x 5}-3 g_{234} e_{z 1}+3 \gamma_{13} g_{234} e_{z 3}\right)+2(1+\alpha) c_{44}\left(-h_{35}+g_{53} e_{z 3} / k_{33}\right. \\
& \left.\left.+\frac{g_{135}}{k_{33} k_{55}}\left(k_{33} e_{51}-k_{135} e_{z 3}\right)\right)\right], \\
C_{030}= & \frac{1}{2}(1+3 \alpha)\left(c_{22}-\beta_{23} c_{234}\right)+\alpha^{2}\left[2 \alpha \kappa_{44} k_{22}-3\left(\gamma_{23}\left(c_{44}-k_{23}\right)-k_{22}\right)\right] \\
& -\frac{\alpha^{2} \kappa_{44}}{h_{33}}\left[2 k_{44} h_{34}(1+\alpha)+\frac{e_{z 3}}{k_{33}}\left(-2 g_{43} k_{44}(1+\alpha)+g_{234}\left(2 k_{234}(1+\alpha)-3 \alpha k_{23}\right)\right)\right. \\
& \left.+2 \alpha k_{23} h_{234}+g_{234}\left(\alpha e_{z 2}-2 e_{42}(1+\alpha)\right)\right] .
\end{aligned}
$$

\section{REFERENCES}

[1] S.V. Gopinathan, V.V. Varadan, and V.K. Varadan. A review and critique of theories for piezoelectric laminates. Smart Mater. Struct., 9:24-48, 2000.

[2] J. Wang and J. Yang. Higher-order theories of piezoelectric plates and applications. Appl. Mech. Rev., $53: 87-99,2000$.

[3] A. Fernandes and J. Pouget. An accurate modelling of piezoelectric multi-layer plates. Europ. J. Mech. A - Solids, 21:629-651, 2002.

[4] S. Kapuria. A coupled zig-zag third-order theory for piezoelectric hybrid cross-ply plates. J. Appl. Mech., 71:604-614, 2004.

[5] S. Kapuria and G.G.S. Achary. A coupled zigzag theory for the dynamics of piezoelectric hybrid cross-ply plates. Arch. Appl. Mech., 75:42-57, 2005.

[6] D.H. Robbins and I. Chopra. The effect of laminate kinematic assumptions on the global response of actuated plates. J. Int. Mater. Syst. Struct., 17:273-299, 2006.

[7] C.P. Wu and J.Y. Lo. An asymptotic theory for dynamic response of laminated piezoelectric shells. Acta Mech., 183:177-208, 2006.

[8] A. Boström, G. Johansson, and P. Olsson. On the rational derivation of a hierarchy of dynamic equations for a homogeneous, isotropic, elastic plate. Int. J. Solids Struct., 38:2487-2501, 2001.

[9] G. Johansson and A.J. Niklasson. Approximate dynamic boundary conditions for a thin piezoelectric layer. Int. J. Solids Struct., 40:3477-3492, 2003.

[10] B. Zhang, A. Boström, and A.J. Niklasson. Antiplane shear waves from a piezoelectric strip actuator: exact versus effective boundary condition solutions. Smart Mater. Struct., 13:161-168, 2004.

[11] A. Boström and B. Zhang. In-plane P-SV waves from a piezoelectric strip actuator: exact versus effective boundary condition solutions. IEEE Trans. Ultrason. Ferroelectr. Freq. Control, 52:1594$1600,2005$.

[12] B.A. Auld. Acoustic Fields and Waves in Solids. Krieger, Malabar, FL, 1990.

Department of Applied Mechanics, Chalmers University of Technology, SE-41296 GöteBORG, SWEDEN 